\title{
Trajectory reconstruction techniques for evaluation of ATC systems
}

\author{
J. García, Member, IEEE, \\ GIAA, Universidad Carlos III de Madrid \\ A. Soto, G. de Miguel, J. Besada and P. Tarrio \\ GPDS, Universidad Politécnica de Madrid \\ Avda Universidad 22, 28270 Colmenarejo, Spain \\ phone: + (34) 918561315, fax: + (34) 918561271 , email: jgherter(a.inf.uc3m.es
}

\begin{abstract}
This paper is focused on trajectory reconstruction techniques for evaluating ATC systems, using real data of recorded opportunity traffic. We analyze different alternatives for this problem, from traditional interpolation approaches based on curve fitting to our proposed schemes based on modeling regular motion patterns with optimal smoothers. The extraction of trajectory features such as motion type (or mode of flight), maneuvers profile, geometric parameters, etc., allows a more accurate computation of the curve and the detailed evaluation of the data processors used in the ATC centre. Different alternatives will be compared with some performance results obtained with simulated and real data sets.
\end{abstract}

Index Terms - trajectory reconstruction, air traffic control, sensor fusion

\section{INTRODUCTION}

Air Traffic Control (ATC), as a critical application involving safety, requires from validation in real operative conditions. Performance assessment of ATC centers by means of recorded datasets (named opportunity traffic data) requires as a previous step the reconstruction of all the reference information, since there is no ground truth available [1-2]. The evaluation is based on the comparison of the output of a specific sensor or an ATC tracker with the trajectories reconstructed from all available data. Regarding the first aspect, there are diverse systems used for sensor evaluation, which extract data about a specific sensor (primary/secondary radars, ADS, etc.) to analyze its behavior in terms of coverage, probability of detection, missed reports, etc. These systems are generally depending on the sensor itself, usually developed to test and configure the installation. In the case of ATC evaluation, the number of currently operating systems is much more reduced. As a representative example, we can mention the evaluation suite maintained by Eurocontrol, which provides a general set of analysis tools, displays, etc., to make possible an extensive analysis of the global system performance. We will specifically address here the evaluation of surveillance systems based on ATC sensors (primary and secondary radar, aircraft derived data, ADS, wide-area multilateration, etc.).

The reconstruction to estimate the trajectories is performed off-line over stored data, and can be formulated as a special multi-sensor fusion process, named smoothing [3]. The particular aspect is the advantage of knowledge about both past and future target position reports to improve the accuracy of result. While ATC processors must provide the result in real time (data are processed in a single sequence at the same time as measurements are received), the reconstruction is a batch process which makes use of all available data.

Data smoothing has been tackled from different points of view. In non-parametric models, a free equation such as a polynomial curve or a soft-computing system (such as neural network) can be selected as basic shape to model the curve. The curve can be the result of optimization for smoothness, while minimizing error against the sampled data points in both position and velocity. Splines are the most used methods for non-parametric data fitting. For instance, one of the reconstruction techniques currently being operated uses approximation beta-splines [4]. This type of solutions does not exploit knowledge about target motion, but has the advantage of being quite general and robust for cases were accurate models of the motion are not available, for instance they are used for modeling human or terrain robots movement on ground [5-6]. As an alternative, if a parametric representation is a priori known for the motion being modeled (parabolic motion for a falling object, coordinated turn for regular flight motion, etc.), then well established estimation methods may be used. A theoretically optimal approach consists in a double tracking loop in the forward and backward directions for smoothing, named as "optimal smoother' $[3,9,10]$.

The two basic aspects for estimating the reference trajectory are the development of appropriate models for sensor errors and target behavior. The model of sensor errors should address the probability density function (systematic and random components), to be exploited in the reconstruction process. Regarding the model of target behavior, in this work we propose a model-based 
reconstruction taking advantage of physical motion models ad hoc for aircraft flying in controlled airspace. In the case of aircraft flying within regulated airspace, they follow quite regular patterns, although at the same time the system also has to consider more irregular flights in other areas and the eventual presence of military aircraft performing high-energy maneuvers (HEM).

In this study we develop a reconstruction architecture based on these ideas. Several variations in the literature are analyzed to develop approximations to the "optimal smoother" [9-10], taking as benchmark non-parametric methods based on smoothing splines. Comparative results on simulated and real data sets will be discussed to conclude their suitability to this specific domain.

\section{SENSOR ERRORS AND AIRCRAFT-MOTION MODELS}

The reconstruction process has been developed to exploit the models of uncertainty in sensors and aircraft motion with a maximum independence of user. It is a special multisensor fusion system, aiming to estimate target kinematic state, taking advantage of knowledge of future target position reports. First, the preprocessing phase obtains bias-free multisensor associated lists of target reports unique for each trajectory. They must be time/spatially aligned and converted to a common fusion coordinate frame, considering all sensor specific information. Then, the3D reconstruction of target trajectories is based on the associated lists of measures.

\section{A. Sensor error models}

The errors in measurements have been modeled with a constant term, bias, and a random component, with parameters obtained from data. Gaussian distribution is assumed for position measurements and uniform in the case of height, in which barometric altitude is mainly affected by quantification, and the same for navigation airborne-derived data such as groundspeed, heading, vertical speed, etc. In the case of secondary radars, in which the range is computed from the transponder replay, an explicit bias term is added to model its behaviour. Finally, it has been considered the presence of outliers, those data significantly deviated from the rest, which are removed from the reconstruction.

With respect to random components of measurement error, their parameters are computed to be taken into account in the reconstruction algorithms, summarized by noise covariance matrices which will be used in the corresponding Kalman filters of each algorithm. Their main characteristics are:

- Radar. Radar random error is assumed to be of constant standard deviation for all distances, and there are two components, aligned with measurements: Radial noise and azimuth noise. Different radar modes of operation may have different noise variances, they are provided in a mode-by-mode basis per radar by the user.

- ADS. ADS measures random error comes from several problems in the complete ADS system: Navigation system error (GPS or other), parameterized by
NUK/FOM information in the ADS data; quantification error, due to the communication channel limited bandwidth; and time stamping error, due to the lack of synchronism between airborne time reference and ADS ground station.

- WAM. The WAM measurements may have a covariance included, provided for this measurement by the multilateration system. Otherwise, we use an error map, with standard deviations defined by spatial cells.

With respect to bias terms, they are estimated to be subtracted from measurements. In the case of radars, there are some terms which are related to all targets in radar coverage, while some other are only related to each specific target (e.g. transponder delays). The PSR/SSR bias terms is a $6-\mathrm{D}$ vector containing range, range gain, azimuth, azimuth eccentricity (two parameters, magnitude and azimuth with maximum error), and time stamp. Secondary radars include the transponder delay, different for each aircraft. In the case of ADS and WAM, bias terms are expressed as $X-Y$ components, fixed in the case of ADS and distributed in a map for WAM.

\section{B. Aircraft-motion models}

A-priori models for the motion of targets allow the application of well established estimation methods. In our case, the reconstruction is based on the regularity of commercial aircraft flying within predefined airways. The trajectory is segmented in intervals with identified Mode of Flight (MoF): Uniform motion, Transversal manoeuvre, Longitudinal manoeuvre, Combined manoeuvre. Then, an accurate reconstruction can be performed using trajectory interpolation accordingly to the models identified in the flight. The dynamic equations regulating the system behavior can be defined as a collection of operation modes. A first stage identifies which mode is active for every time interval in order to apply the appropriate motion model. In the case that no regular model can be applied, a default reconstruction mode is used which avoids the assumption of specific models and gives higher credibility to the sensor measurements.

\section{MODEL-BASED RECONSTRUCTION}

Since target behavior may change dramatically (e.g. development of maneuvers inserted between uniformmotion segments), a single kinematic model is insufficient. We have applied an Interacting Multiple Model (IMM) algorithm to model the aircraft behavior, a well referred algorithm for maneuvering target tracking which dynamically determines the most likely model at each time [7-8]. This algorithm has been extensively applied to a type of problems referred to as jump Markov or hybrid-state problem, which involves the joint estimation both for the continuous-valued state vector and a discrete-valued state variable, based only in the sequence of available observations. In our case, the state vector contains position and velocity in reconstruction $\vec{x}[k]=\left[\begin{array}{llll}\hat{x}[k] & \hat{v}_{x}[k] & \hat{y}[k] & \hat{v}_{y}[k]\end{array}\right]^{t}$, while discrete 
variable $\theta[\mathrm{k}]$ represents the $\mathrm{MoF}$ at each time, $\{\mathrm{U}, \mathrm{T}, \mathrm{L}\}$, denoting Uniform, Transversal, Longitudinal.

Our approach carries out two stages. The trajectory is segmented in regular motion segments with a first pass to extract the segments. Then, in the second phase, an accurate reconstruction, with less uncertainty, can be performed accordingly to the models identified in the flight and actively taking into account the transitions and parameters identified.

The segments are obtained from the IMM mode probabilities computed in a double forward-backward loop. This process provides a first division of sequence of associated measures in $\mathrm{MoF}$ segments, but probably with a high rate of over-division produced by noisy transitions. So, a refinement is applied in a second phase. Very short segments are removed, fused with neighbor adjacent segments, and adjacent uniform segments are analyzed to check if they can be fused. Finally, all MoF segments are validated with a least-squares test. The kinematic parameters corresponding to different types of motion are computed to generate idealized paths, and then the averaged normalized residual is compared with a maximum threshold. If the averaged residual is not acceptable, the segment is labeled as "unknown".

Then, the trajectory interpolation is carried out with an optimal smoothing filter operating forward and backward [3]. It takes into account the advantage of classified $\mathrm{MoF}$ segments when available and validated (those accomplishing the least-squares fitting test). The maneuvering parameters describing the "mean" values along the segment are used in the dynamic models, adapted to the specific conditions of the data segment, as displayed in figure 1. For instance, in the case of turn model, a circular prediction model is applied, taking into account the parameters for circle radius and centre. Besides, the information about motion intervals is used in the structure. The transition probabilities are modified in the intervals close to edges, and the plant noises are also increased in the presence of close transitions. When no validated segment class is available ("unknown" or "recovered" segment), the prediction filters uses default values and a wider plant noise is used to avoid degradation. Therefore, all modes exploit information about the parameters used in reconstruction and also if they are applicable or not.

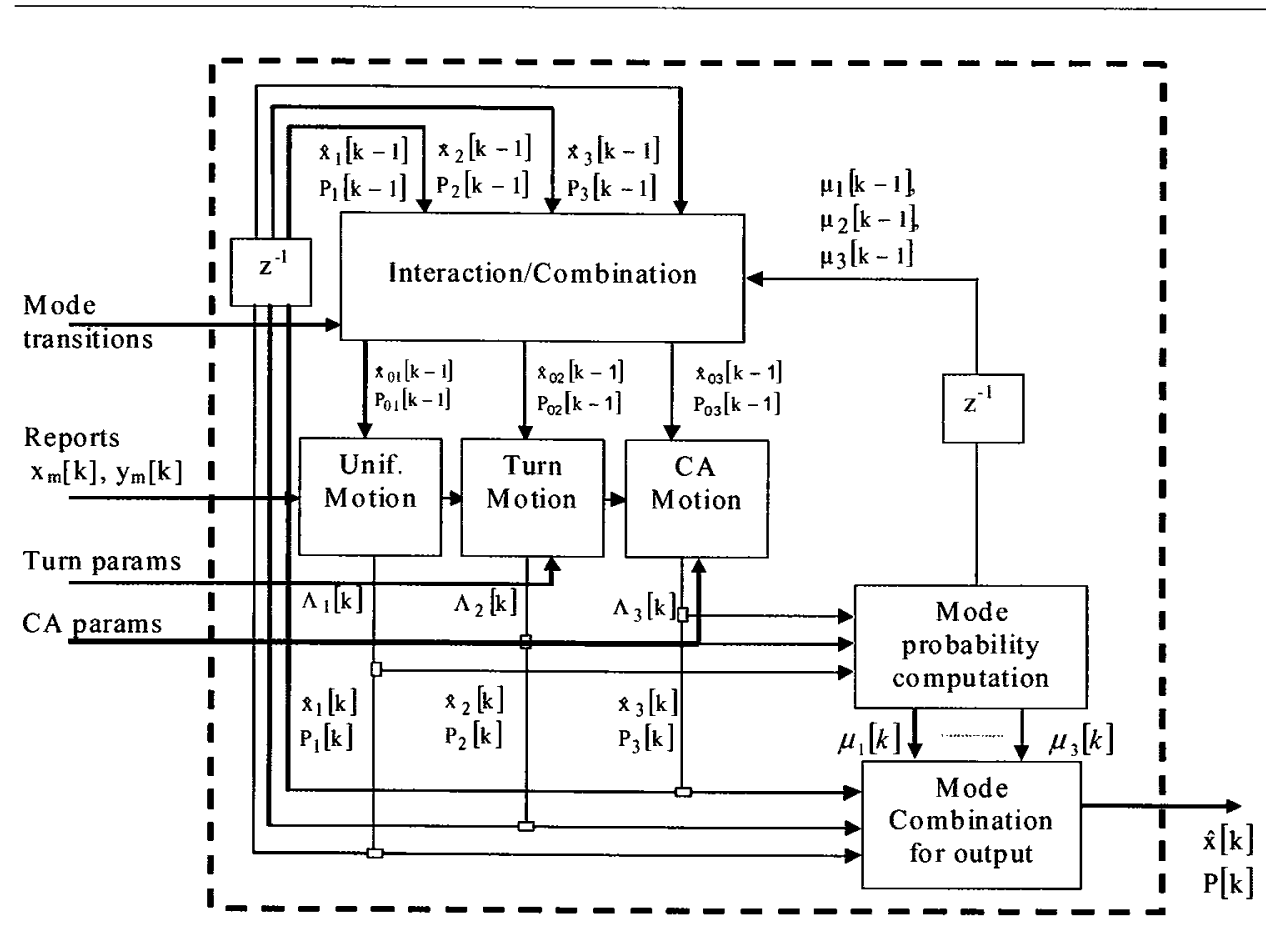

Figure 1 -IMM parametric reconstruction filter 
If available Aircraft Derived Data (ADD), the velocity data in the target reports from Mode-S and ADS sensors are also used in the reconstruction. In this case, the velocity measurements (groundspeed and heading), are projected onto stereographic plane, together with their error covariance matrix, and integrated in the reconstruction filters through variable-size projection matrices. In order to avoid instabilities due to bad information, a consistency check is performed before integrating this velocity information in the reconstruction. The deviation between estimated and measured velocities is compared with a maximum allowed deviation before acceptance.

\section{OTHER Previous APproaches}

In order to assess the output of the Model-based algorithm, two other different reconstruction techniques have been taken into account. The idea is to show the advantages and disadvantages of this new reconstruction approach compared with two classical ones. One of them is a suboptimal fixedinterval smoothing algorithm presented by Helmick et al [9] and the other one is a spline-based reconstruction. In the following subsections both techniques are going to be described in more detail.

\section{A. Fixed-interval smoother}

This is a suboptimal approach based on the use of two IMM filters [9]. One of the filters propagates in the forward-time direction and the other one in the backward-time direction, which is quite similar to what is done by the Model-based approach in order to obtain the MoF segments.

This algorithm is an approximation that considers only the motion model over two successive sampling periods. Thus, the implemented version consists of two IMM filters, one forward and one backward, with two motion models implemented. Both of them are constant velocity models, one with no plant noise $\left(Q_{1}=0\right)$ and the other with plant noise covariance:

$$
Q_{2}=\left[\begin{array}{cccc}
T^{4} / 4 & T^{3} / 2 & 0 & 0 \\
T^{3} / 2 & T^{2} & 0 & 0 \\
0 & 0 & T^{4} / 4 & T^{3} / 2 \\
0 & 0 & T^{3} / 2 & T^{2}
\end{array}\right]
$$

The initial model probabilities in the forward-time filter are $\mu_{0}=\left[\begin{array}{ll}0.9 & 0.1\end{array}\right]^{\mathrm{T}}$ and the model-switching probability matrix is given by:

$$
P_{i j}=\left[\begin{array}{ll}
0.95 & 0.05 \\
0.10 & 0.90
\end{array}\right]
$$

The final measurement and its covariance are used to obtain initial position estimates and covariance in the backward-time filter. For this filter, the initial mode probabilities have been chosen to be $\mu_{\mathrm{T}}=\left[\begin{array}{ll}0.5 & 0.5\end{array}\right]^{\mathrm{T}}$.

\section{B. Splines}

The second reconstruction technique is based on a "natural" cubic spline which is directly implemented using a MATLAB function (csaps). This second comparison is provided in order to show the behavior of a non-parametric data fitting with no special tuning.

The results obtained using the three techniques are going to be shown in the following section.

\section{Simulations}

In this section, the reconstructions performed using the previously described techniques are going to be presented. Two synthetic scenarios and two real ones have been chosen in order to assess the quality of the reconstruction in different representative scenarios. For the sake of clarity, only the ideal trajectory, in the synthetic scenarios, is going to be depicted omitting the output of each method. After that, two figures representing the absolute error will show the performance of the different methods. For the real scenarios, as there is no ground truth it has been chosen to show the measurements and the output of the Model Based reconstruction. In order to analyze more in depth, several manoeuvres have been zoomed in which allows to see the quality of the reconstruction.

\section{A. Synthetic scenarios}

The first synthetic scenario is the following one:

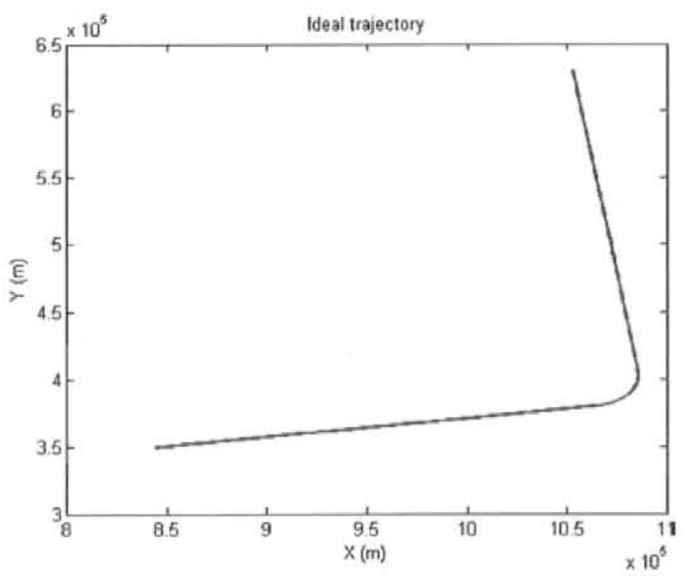

Figure 2 - Ideal trajectory

It consists of an aircraft that performs a left turn after a straight segment which is continued by another straight segment. It goes from the west to the east at a constant velocity of $300 \mathrm{~m} / \mathrm{s}$.

The absolute position error in the reconstruction is shown in the two following figures. As it can be seen, the output of the parametric approaches is better than the one provided by the spline one. In can be seen that the output of the Model-based approach has less error peak than the fixed-interval smoother. 
If available Aircraft Derived Data (ADD), the velocity data in the target reports from Mode-S and ADS sensors are also used in the reconstruction. In this case, the velocity measurements (groundspeed and heading), are projected onto stereographic plane, together with their error covariance matrix, and integrated in the reconstruction filters through variable-size projection matrices. In order to avoid instabilities due to bad information, a consistency check is performed before integrating this velocity information in the reconstruction. The deviation between estimated and measured velocities is compared with a maximum allowed deviation before acceptance.

\section{OTHER Previous APPRoACHES}

In order to assess the output of the Model-based algorithm, two other different reconstruction techniques have been taken into account. The idea is to show the advantages and disadvantages of this new reconstruction approach compared with two classical ones. One of them is a suboptimal fixedinterval smoothing algorithm presented by Helmick et al [9] and the other one is a spline-based reconstruction. In the following subsections both techniques are going to be described in more detail.

\section{A. Fixed-interval smoother}

This is a suboptimal approach based on the use of two IMM filters [9]. One of the filters propagates in the forward-time direction and the other one in the backward-time direction, which is quite similar to what is done by the Model-based approach in order to obtain the $\mathrm{MoF}$ segments.

This algorithm is an approximation that considers only the motion model over two successive sampling periods. Thus, the implemented version consists of two IMM filters, one forward and one backward, with two motion models implemented. Both of them are constant velocity models, one with no plant noise $\left(Q_{1}=0\right)$ and the other with plant noise covariance:

$$
Q_{2}=\left[\begin{array}{cccc}
T^{4} / 4 & T^{3} / 2 & 0 & 0 \\
T^{3} / 2 & T^{2} & 0 & 0 \\
0 & 0 & T^{4} / 4 & T^{3} / 2 \\
0 & 0 & T^{3} / 2 & T^{2}
\end{array}\right]
$$

The initial model probabilities in the forward-time filter are $\mu_{0}=\left[\begin{array}{ll}0.9 & 0.1\end{array}\right]^{\mathrm{T}}$ and the model-switching probability matrix is given by:

$$
P_{i j}=\left[\begin{array}{ll}
0.95 & 0.05 \\
0.10 & 0.90
\end{array}\right]
$$

The final measurement and its covariance are used to obtain initial position estimates and covariance in the backward-time filter. For this filter, the initial mode probabilities have been chosen to be $\mu_{\mathrm{T}}=\left[\begin{array}{ll}0.5 & 0.5\end{array}\right]^{\mathrm{T}}$.

\section{B. Splines}

The second reconstruction technique is based on a "natural" cubic spline which is directly implemented using a MATLAB function (csaps). This second comparison is provided in order to show the behavior of a non-parametric data fitting with no special tuning.

The results obtained using the three techniques are going to be shown in the following section.

\section{Simulations}

In this section, the reconstructions performed using the previously described techniques are going to be presented. Two synthetic scenarios and two real ones have been chosen in order to assess the quality of the reconstruction in different representative scenarios. For the sake of clarity, only the ideal trajectory, in the synthetic scenarios, is going to be depicted omitting the output of each method. After that, two figures representing the absolute error will show the performance of the different methods. For the real scenarios, as there is no ground truth it has been chosen to show the measurements and the output of the Model Based reconstruction. In order to analyze more in depth, several manoeuvres have been zoomed in which allows to see the quality of the reconstruction.

\section{A. Synthetic scenarios}

The first synthetic scenario is the following one:

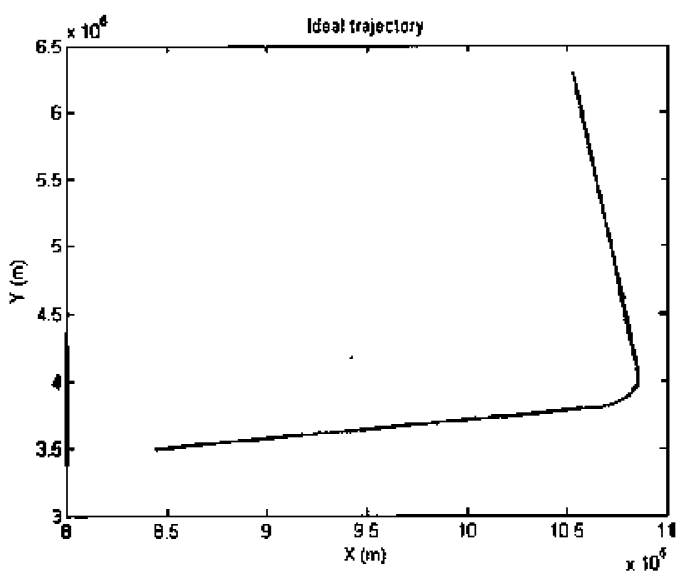

Figure 2 - Ideal trajectory

It consists of an aircraft that performs a left turn after a straight segment which is continued by another straight segment. It goes from the west to the east at a constant velocity of $300 \mathrm{~m} / \mathrm{s}$.

The absolute position error in the reconstruction is shown in the two following figures. As it can be seen, the output of the parametric approaches is better than the one provided by the spline one. In can be seen that the output of the Model-based approach has less error peak than the fixed-interval smoother. 


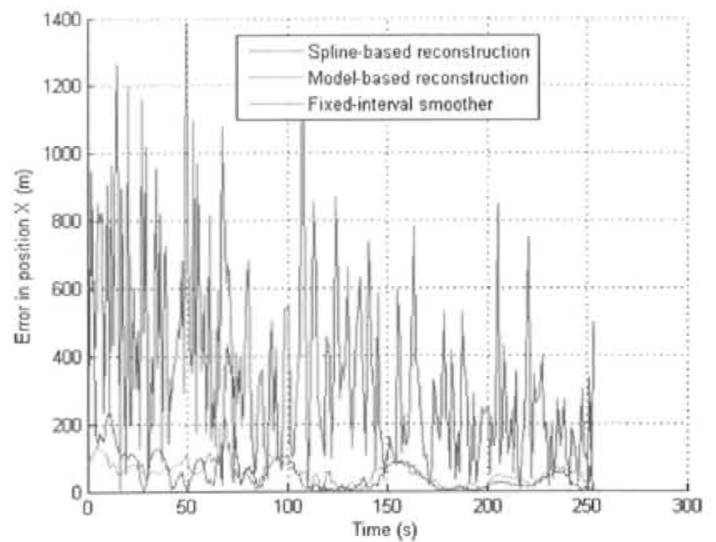

Figure 3 - Error in position $\mathrm{X}$

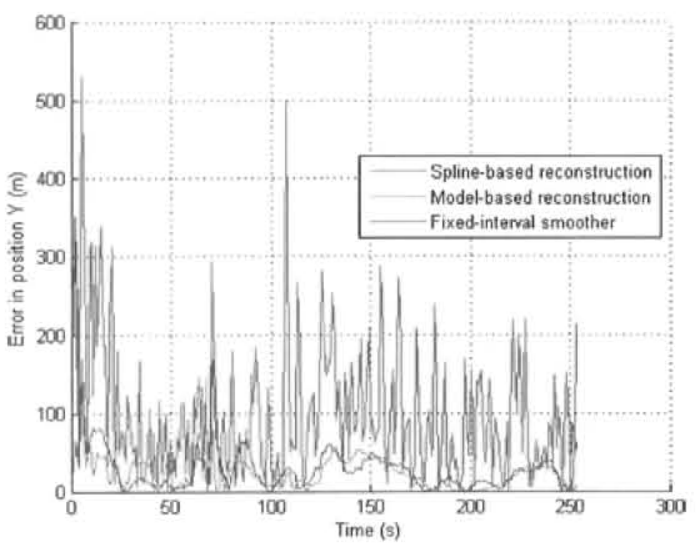

Figure 4 - Error in position $Y$

The second synthetic scenario is:

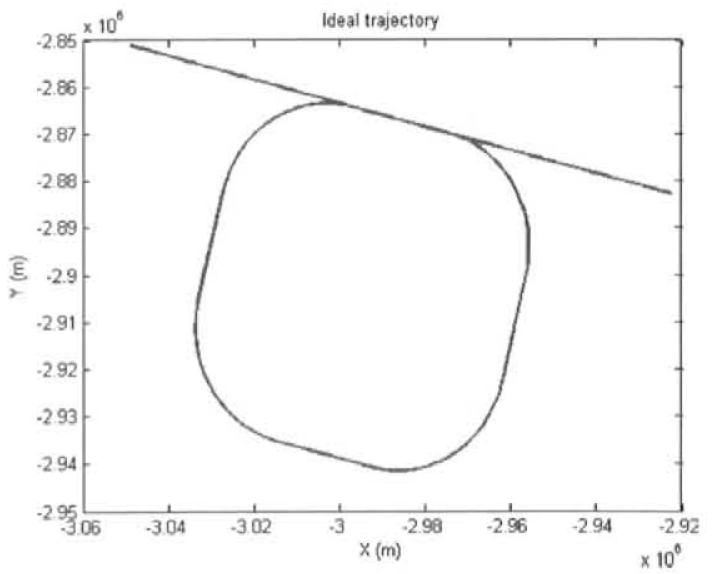

Figure 5-Ideal trajectory

The aircraft goes also from west to east at a velocity of around $300 \mathrm{~m} / \mathrm{s}$ performing a loop as if it was waiting for landing in a certain airport. Next, the following figures show the error made in the reconstruction.

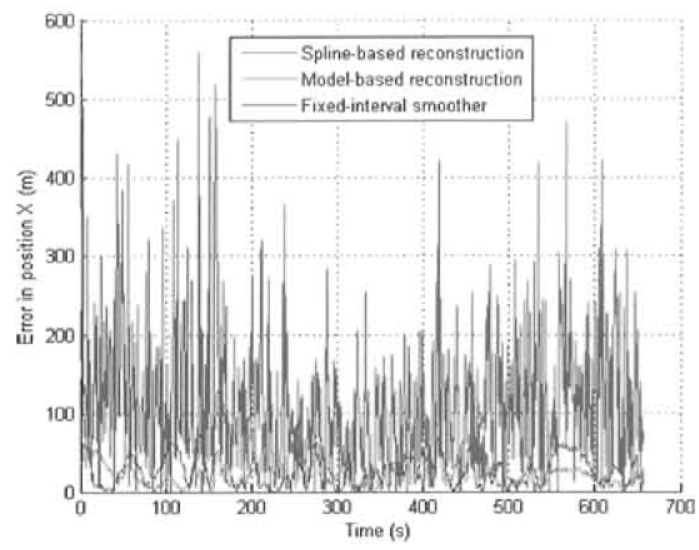

Figure 6 - Error in position $\mathrm{X}$

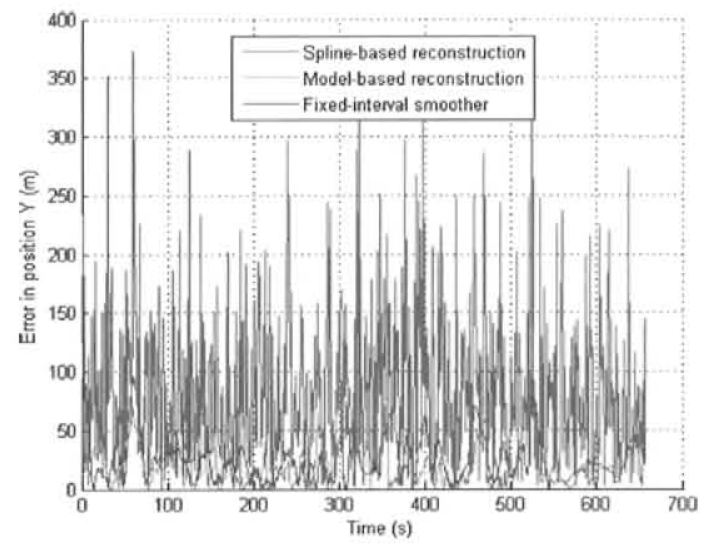

Figure 7 - Error in position Y

\section{B. Real scenarios}

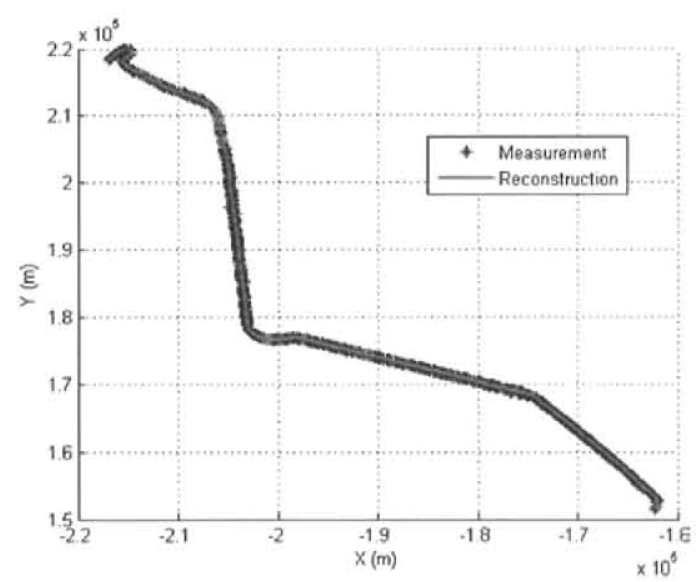

Figure 8-Measurements and reconstructed trajectory "A" 


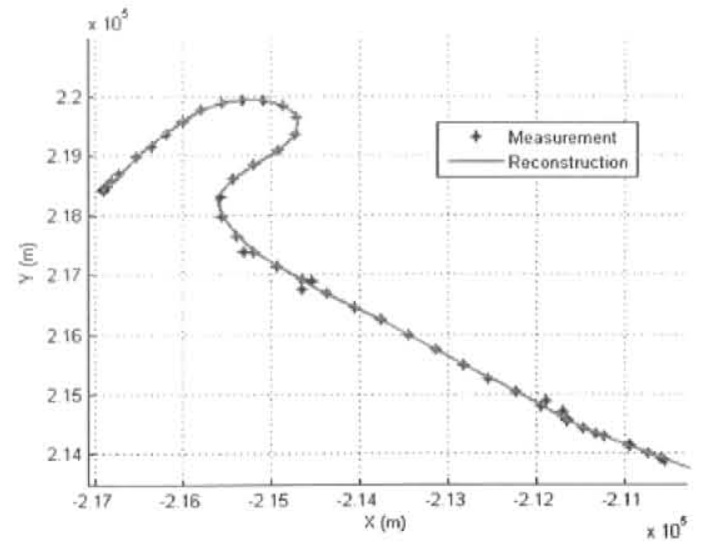

Figure 9 - Reconstruction detail of trajectory "A"

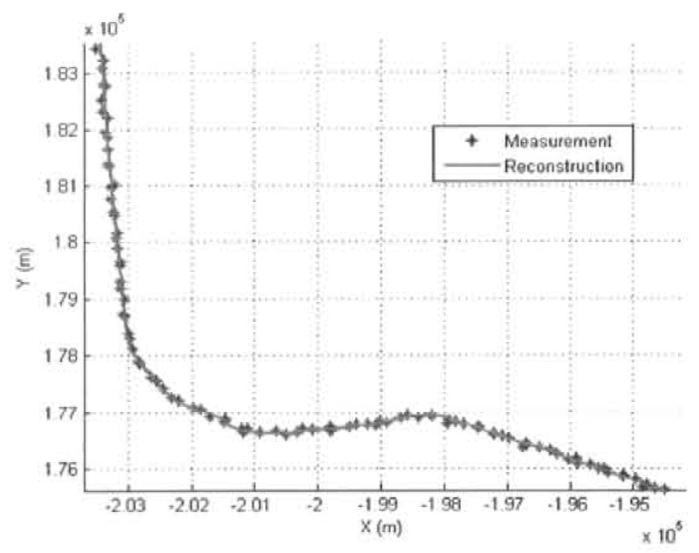

Figure 10-Reconstruction detail of trajectory " $A$ "

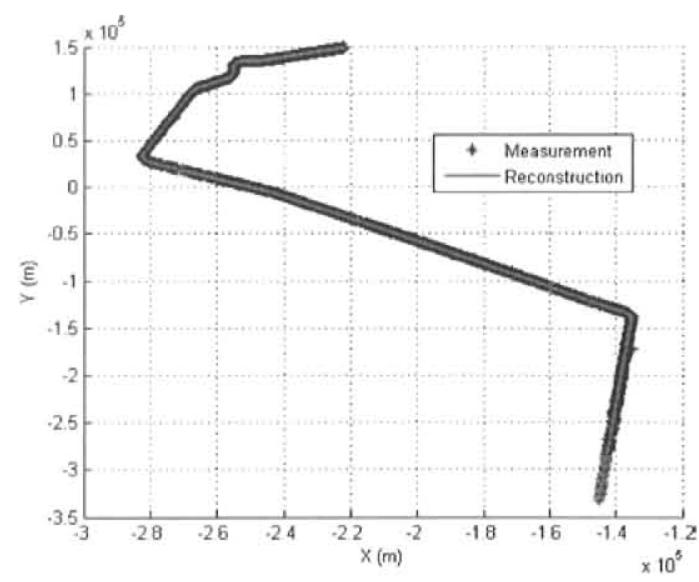

Figure 11 - Measurements and reconstructed trajectory "B"

As can be seen, the quality of the reconstruction in real scenarios is more than reasonable, being robust enough to cope with outliers as can be observed in figure 12 . However, the reconstruction can be improved by parameter tuning. Which implies a trade-off between noise reduction and manoeuvre response.

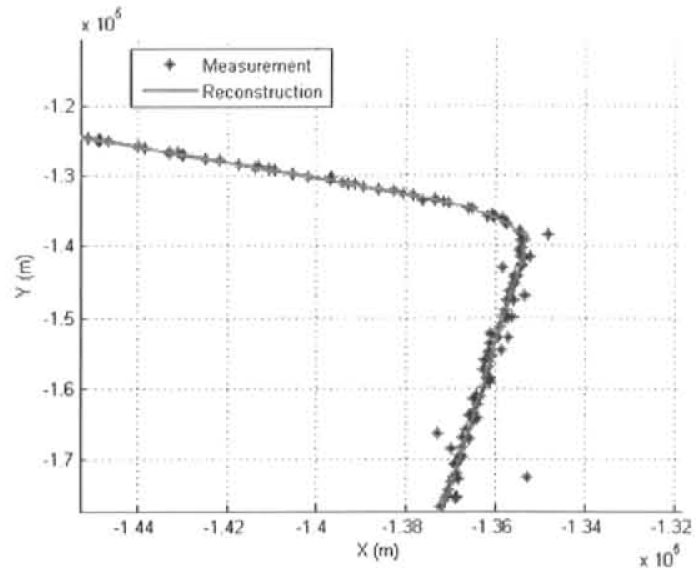

Figure 12 - Reconstruction detail of trajectory " $B$ "

\section{SUMMARY}

Alternative approaches for trajectory reconstruction have been proposed and analyzed. Model-based reconstruction takes advantage of the presence of regular motion patterns in ATC flights. Its performance was compared with a conventional data fitting process based on splines and a fixed-interval smoother, both in simulated and real representative scenarios. The availability of kinematic on-board measurements allowed and additional improvement of accuracy both in segmentation and interpolation performance.

\section{REFERENCES}

[1] J.J. Renes, P.v.d. Kraan, C. Eymann, "Flightpath reconstruction and systematic radar error estimation from multi-radar range-azimuth measurements", Proceedings of 24th IEEE Conference on Decision and Control, 1985

[2] A. Desmond-Kennedy, B. Gardner, "Tools for analysing the performance of ATC surveillance radar"s, IEE Colloquium on Specifying and Measuring Performance of Modem Radar Systems, March 1998.

[3] A. Gelb, "Applied optimal estimation", The M.I.T. Press, July 1982.

[4] J.J. Renes, M.R. Best, "MURATREC Multi-Radar trajectory reconstruction facility theoretical background", NLR CR 89196 L, National Lucht- en Ruimtevaartlaboratorium.

[5] S. Takahashi, C. F. Martin, "Optimal control theoretic splines and its application to mobile robot". Proceedings of the 2004 IEEE International Conference on Control Applications, Sept. 2004.

[6] J. Aleotti, S. Caselli, G. Maccherozzi, "Trajectory Reconstruction with NURBS Curves for Robot Programming by Demonstration", Proceedings of the 2005 IEEE International Symposium on Computational Intelligence in Robotics and Automation, June, 2005.

[7] Y.Bar-Shalom, Ed., "Multitarget-Multisensor Tracking: Advanced Applications". Norwood, MA: Artech House, 1990.

[8] X. Rong Li, Y. Bar-Shalom, "Design of an Interacting Multiple Model Algorithm for Air Traffic Control Tracking", IEEE Transactions on Control System Technology, vol. 1, No. 3, September 1993.

[9] R. E. Helmick, W. D. Blair, S. A. Hoffman, "Fixed-Interval Smoothing for Markovian Switching Svstems", IEEE Transactions on Information Theory, vol. 41, No. 6, November 1995.

[10] J. García, J.M. Molina, J. Besada, G. de Miguel, "Model-Based Trajectory Reconstruction using IMM Smoothing and Motion Pattern Identificacion", Proceedings of the $10^{\text {th }}$ International Conference on Information Fusion, 2007. 